\title{
Aggregation Kinetics and Stability Mechanisms of Pristine and Oxidized \\ Nanocarbons in Polar Solvents (Supporting Information)
}

\author{
Nima Sefidmooye A zar ${ }^{\dagger}$ and Mahdi Pourfath*,+,,, II \\ School of Electrical and Computer Engineering, University of Tehran, Tehran, Iran, School of Nano \\ Science, Institute for Research on Fundamental Sciences (IPM), P.O.Box 19395-5531, Tehran, Iran, \\ and Institute for Microelectronics, Technische Universität Wien, Vienna 1040, Austria \\ Tel: +98-21-6111-4982, Fax: +98-21-8801-3199 \\ E-mail: pourfath@ut.ac.ir;pourfath@iue.tuwien.ac.at
}

\footnotetext{
*To whom correspondence should be addressed

${ }^{\dagger}$ University of Tehran

‡IPM

ITTechnische Universität Wien
} 


\section{Calculation of the Nanocarbons Partial Charges}

In the case of a large nanocarbon, which only contains unpolarized carbon-carbon bonds, the atoms can be safely considered uncharged in classical molecular dynamics simulations. However, here we used finite-size hydrogen-terminated nanocarbons. DFT calculations showed an average charge with a magnitude of $\sim 0.14$ e on the end hydrogens and the carbon atoms attached to them. If these partial charges are taken into account, they will put unnecessary emphasis on the small size of the nanocarbons. Our studies showed that considering these partial charges adds a negligibly small repulsive component to the PMF profiles. Consequently, all basal carbon atoms (except for the ones bonded to the functional groups in the functionalized structures) as well as the end hydrogens were treated as uncharged atoms in this study.

In order to calculate the partial charges on carboxyl and hydroxyl functional groups as well as the basal carbon atoms bonded to them, the two sample structures illustrated in Figure S2 were considered. These structures were optimized employing the B3LYP/6$311++G^{* *}$ method and the partial charges were calculated using the same level of theory. Subsequently, the very small differences in the partial charges on the identical atoms were filtered out by averaging the values. Also the partial charges were truncated to the nearest neighbor of the basal carbon atom covalently bonded to the functional group. The results are depicted in Figure $\mathrm{S3}$ and Table $\mathrm{S10}$ and $\mathrm{S11}$.

It should also be mentioned that the structural parameters such as bond lengths and angles of these DFT-optimized samples were compared to the results obtained from the SCC-DFTB approach which was utilized to optimize the nanocarbons in the main simulations. This showed that the results obtained from the much faster SCC-DFTB approach are in good consistency with the ab initio calculations. 


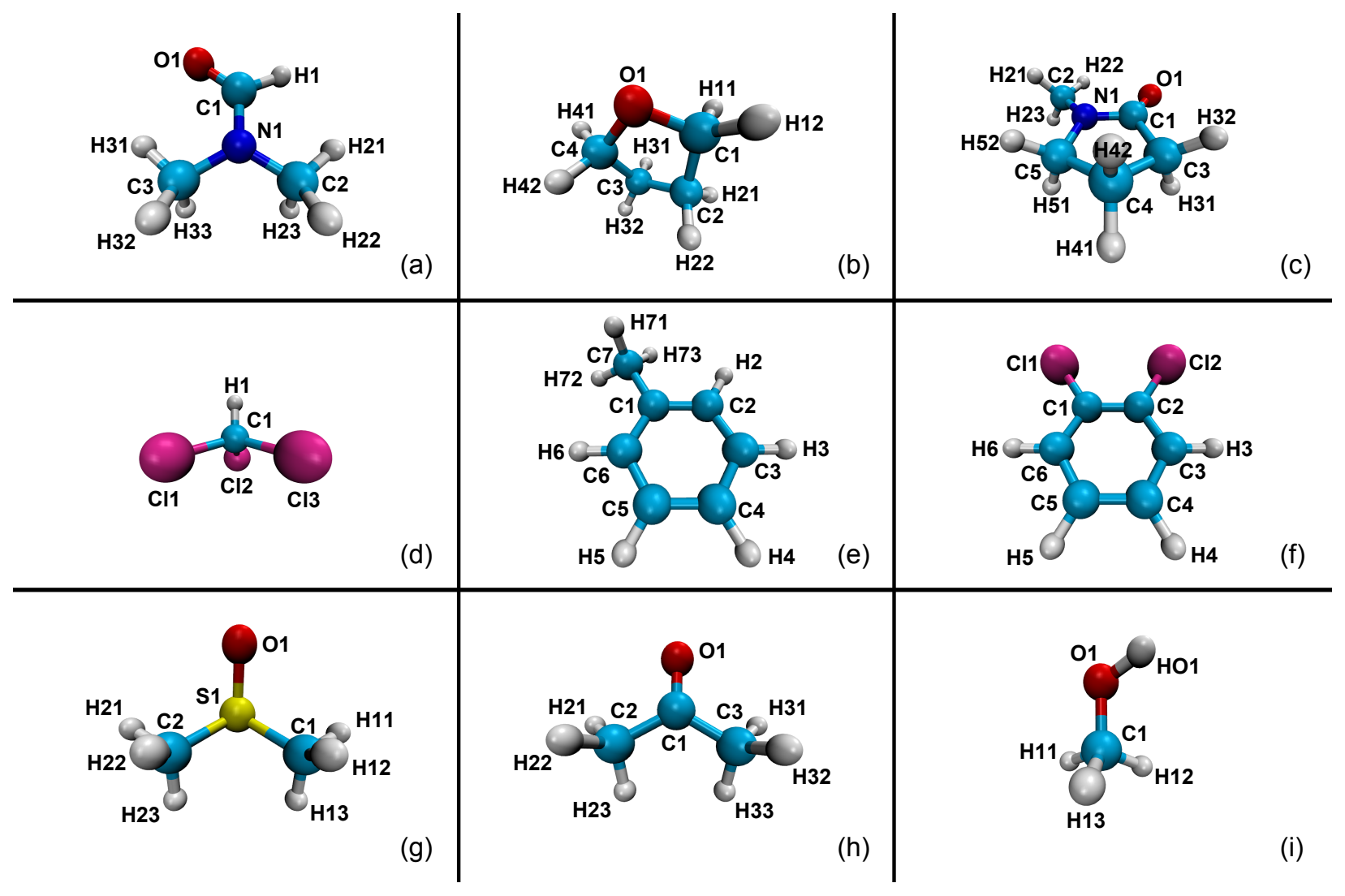

Figure S1: The atom labels used in the following tables for (a) dimethylformamide (DMF), (b) tetrahydrofuran (THF), (c) N-methyl-2-pyrrolidone (NMP), (d) chloroform, (e) toluene, (f) 1,2-dichlorobenzene (ODCB), (g) dimethyl sulfoxide (DMSO), (h) acetone, and (i) methanol. Each sphere represents an atom using this color code: light blue, carbon; white, hydrogen; red, oxygen; dark blue, nitrogen; yellow, sulfur; violet, chlorine.

Table S1: Partial charges of DMF obtained from DFT calculations

\begin{tabular}{cc}
\hline Atom & Partial charge (e) \\
\hline C1 & 0.301 \\
C2 & -0.201 \\
C3 & 0.074 \\
O1 & -0.523 \\
N1 & -0.002 \\
H1 & 0.053 \\
H21 & 0.073 \\
H22 & 0.082 \\
H23 & 0.082 \\
H31 & 0.053 \\
H32 & 0.004 \\
H33 & 0.004 \\
\hline
\end{tabular}


Table S2: Partial charges of THF obtained from DFT calculations

\begin{tabular}{cc}
\hline Atom & Partial charge (e) \\
\hline C1 & 0.356 \\
C2 & 0.021 \\
C3 & 0.021 \\
C4 & 0.356 \\
O1 & -0.530 \\
H11 & -0.041 \\
H12 & -0.042 \\
H21 & -0.030 \\
H22 & 0.001 \\
H31 & 0.001 \\
H32 & -0.030 \\
H41 & -0.042 \\
H42 & -0.041 \\
\hline
\end{tabular}

Table S3: Partial charges of NMP obtained from DFT calculations

\begin{tabular}{cc}
\hline Atom & Partial charge $(\mathrm{e})$ \\
\hline C1 & 0.512 \\
C2 & 0.011 \\
C3 & -0.081 \\
C4 & -0.072 \\
C5 & 0.052 \\
O1 & -0.604 \\
N1 & -0.127 \\
H21 & 0.011 \\
H22 & 0.078 \\
H23 & 0.015 \\
H31 & 0.048 \\
H32 & 0.047 \\
H41 & 0.034 \\
H42 & 0.024 \\
H51 & 0.020 \\
H52 & 0.032 \\
\hline
\end{tabular}

Table S4: Partial charges of chloroform obtained from DFT calculations

\begin{tabular}{cc}
\hline Atom & Partial charge (e) \\
\hline C1 & 0.240 \\
Cl1 & -0.117 \\
Cl2 & -0.117 \\
Cl3 & -0.117 \\
H1 & 0.111 \\
\hline
\end{tabular}


Table S5: Partial charges of toluene obtained from DFT calculations

\begin{tabular}{cc}
\hline Atom & Partial charge (e) \\
\hline C1 & 0.200 \\
C2 & -0.231 \\
C3 & -0.061 \\
C4 & -0.109 \\
C5 & -0.061 \\
C6 & -0.231 \\
C7 & -0.077 \\
H2 & 0.118 \\
H3 & 0.089 \\
H4 & 0.083 \\
H5 & 0.089 \\
H6 & 0.118 \\
H71 & 0.021 \\
H72 & 0.026 \\
H73 & 0.026 \\
\hline
\end{tabular}

Table S6: Partial charges of ODCB taken from the OPLS-AA force field

\begin{tabular}{cc}
\hline Atom & Partial charge (e) \\
\hline C1 & 0.180 \\
C2 & 0.180 \\
C3 & -0.115 \\
C4 & -0.115 \\
C5 & -0.115 \\
C6 & -0.115 \\
C11 & -0.180 \\
C12 & -0.180 \\
H3 & 0.115 \\
H4 & 0.115 \\
H5 & 0.115 \\
H6 & 0.115 \\
\hline
\end{tabular}


Table S7: Partial charges of DMSO taken from the OPLS-AA force field

\begin{tabular}{cc}
\hline Atom & Partial charge (e) \\
\hline C1 & -0.020 \\
C2 & -0.020 \\
O1 & -0.459 \\
S1 & 0.139 \\
H11 & 0.060 \\
H12 & 0.060 \\
H13 & 0.060 \\
H21 & 0.060 \\
H22 & 0.060 \\
H23 & 0.060 \\
\hline
\end{tabular}

Table S8: Partial charges of acetone taken from the OPLS-AA force field

\begin{tabular}{cc}
\hline Atom & Partial charge (e) \\
\hline C1 & 0.470 \\
C2 & -0.180 \\
C3 & -0.180 \\
O1 & -0.470 \\
H21 & 0.060 \\
H22 & 0.060 \\
H23 & 0.060 \\
H31 & 0.060 \\
H32 & 0.060 \\
H33 & 0.060 \\
\hline
\end{tabular}

Table S9: Partial charges of methanol taken from the OPLS-AA force field

\begin{tabular}{cc}
\hline Atom & Partial charge (e) \\
\hline C1 & 0.145 \\
O1 & -0.683 \\
HO1 & 0.418 \\
H11 & 0.040 \\
H12 & 0.040 \\
H13 & 0.040 \\
\hline
\end{tabular}



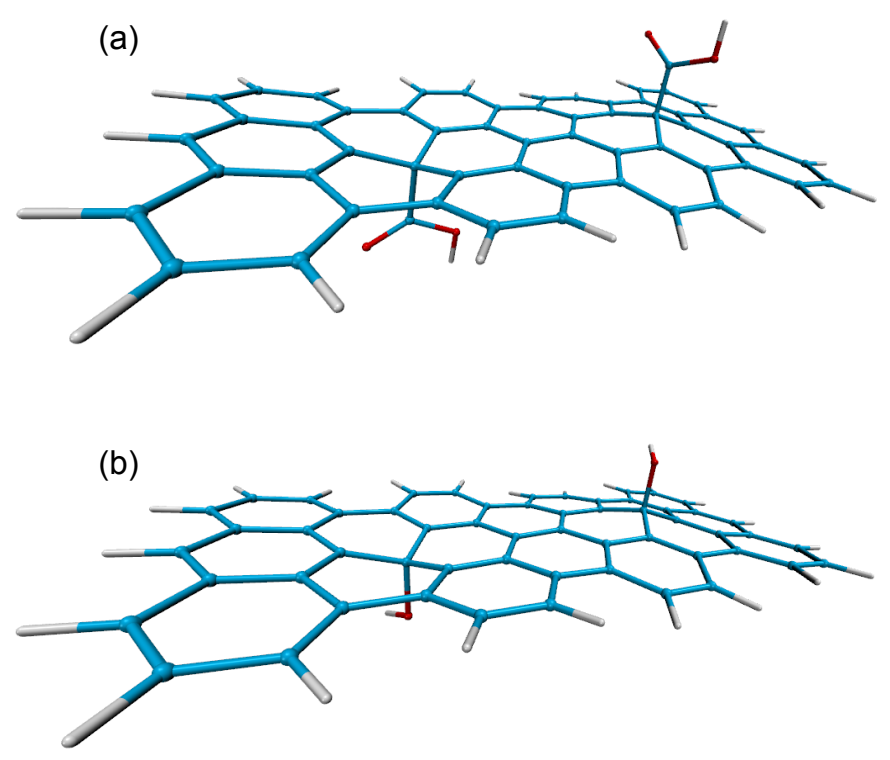

Figure S2: The sample nanocarbon structures optimized through B3LYP/6-311++G** calculations and used for partial charge calculations of the (a) carboxyl and (b) hydroxyl functional groups. color code: light blue, carbon; white, hydrogen; and red, oxygen.

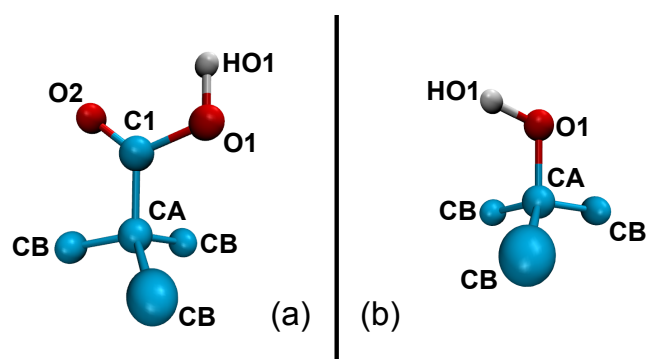

Figure S3: The atom labels used in the following tables for (a) carboxyl and (b) hydroxyl functional groups. color code: light blue, carbon; white, hydrogen; and red, oxygen. CA represents the basal carbon atom which is covalently bonded to the functional group and $\mathrm{CB}$ atoms are the basal nearest neighbors of $\mathrm{CA}$.

Table S10: Partial charges of carboxyl functional group obtained from DFT calculations

\begin{tabular}{cc}
\hline Atom & Partial charge $(\mathrm{e})$ \\
\hline $\mathrm{C} 1$ & 0.829 \\
$\mathrm{O} 1$ & -0.685 \\
$\mathrm{O} 2$ & -0.530 \\
$\mathrm{HO} 1$ & 0.434 \\
$\mathrm{CA}$ & -0.141 \\
$\mathrm{CB}$ & 0.031 \\
\hline
\end{tabular}


Table S11: Partial charges of hydroxyl functional group obtained from DFT calculations

\begin{tabular}{cc}
\hline Atom & Partial charge (e) \\
\hline O1 & -0.685 \\
HO1 & 0.405 \\
CA & 0.718 \\
CB & -0.146 \\
\hline
\end{tabular}

